\title{
Hypoxic Isolated Abdominal Perfusion (HAP) chemotherapy for non-operable advanced staged ovarian cancer with peritoneal carcinosis: an experience in $\mathbf{4 5}$ platinum-refractory ovarian cancer patients
}

\author{
Karl Reinhard Aigner $^{1}$ (D) $\cdot$ Emir Selak $^{1} \cdot$ Sabine Gailhofer $^{1} \cdot$ Kornelia Aigner $^{1}$
}

Received: 18 January 2019 / Accepted: 2 April 2019 / Published online: 16 April 2019

(C) The Author(s) 2019

\begin{abstract}
In order to break through drug resistance in platinum-refractory ovarian cancer, augmented drug exposure was administered to the abdomen by means of an isolated perfusion system. Four cycles of isolated hypoxic abdominal perfusion with cisplatin, adriamycin, and mitomycin were conducted in 4-week intervals. Cisplatin and adriamycin were chosen because of their increased cytotoxicity under hypoxic conditions. Chemofiltration was performed for prophylaxis of cumulative toxicity of adriamycin and mitomycin. The study included 45 patients with recurrent epithelial ovarian cancer who had prior platinum containing therapies (3, stage Federation of Gynecology and Obstetrics (FIGO) IIIB; 20, stage FIGO IIIC; 22; stage FIGO IV). The median survival rate in stage FIGO IIIBC was 12 months, and in stage IV was 10 months. The tumor marker decreased to complete response or partial response at $17.8 \%$ and $55.6 \%$ of the patients. CT or MRI visualization showed complete response in $4.1 \%$, and partial response was in $54.1 \%$. Complete resolution of ascites was noted in $30 \%$ of cases and substantial reduction in another $43 \%$. Toxicity was generally low. Quality of life was improved in the majority of cases. Bone-marrow suppression ranged between WHO grade 1 and 2, and in patients with previous third- or fourth-line chemotherapy, it was WHO grade 3. Isolated hypoxic abdominal perfusion with chemofiltration for patients with progressive and platinum-refractory stage III and IV ovarian cancer is an effective therapy, breaking through chemoresistance and offering comparably long survival at good quality of life.
\end{abstract}

Keywords Ovarian cancer · Quality of life · Chemoresistance · Isolated abdominal perfusion · Intra-arterial chemotherapy

\section{Background}

Ovarian cancer is the leading cause of death among all gynecological cancers. The standard therapy option is still complete cytoreduction when possible, combined with chemotherapy

Karl Reinhard Aigner

info@prof-aigner.de

Emir Selak

e.selak@medias-klinikum.de

Sabine Gailhofer

s.gailhofer@medias-klinikum.de

Kornelia Aigner

kornelia.aigner@medias-klinikum.de

1 Department of Surgical Oncology, Medias Klinikum GmbH \& Co KG, Krankenhausstr. 3a, 84489 Burghausen, Germany
[1-3]. Despite initial response rates of $70-80 \%$ to platinumbased combination chemotherapies with taxanes, platinumresistant recurrences occur very frequently within 2 years. The shorter the recurrence-free interval, the lower the prospect of a renewed response to chemotherapy [4-6]. While increased drug exposure could theoretically lead to a renewed response, they are not universally administered due to increased toxicity [7-15]. Even high-dose or modified combination therapy did not result in any real progress [16-19]. Only recently two randomized studies showed that the addition of HIPEC to cytoreductive surgery resulted in longer recurrence-free and overall survival than cytoreductive surgery alone [20,21].

Alternatively, consideration may be given to new drugs or targeted substances [22-24]. As the response behavior rises sharply with an increased dose or concentration of active agents, we surmised that an isolated extracorporeal perfusion would increase local exposure and break through any existing cytostatic resistance. In order to limit systemic toxicity, which 
could adversely affect quality of life, chemofiltration for detoxification was carried out, following the isolated perfusion procedure $[26,27]$. A case series of 45 heavily pretreated patients with ovarian cancer treated with hypoxic abdominal perfusion is herein reported. It has been applied in patients with advanced and recurrent tumors, resistant to platinumcontaining combination therapies.

\section{Methods}

\section{Patients}

The study included 45 patients at the clinical stages of Federation of Gynecology and Obstetrics (FIGO) IIIB, IIIC, and IV ( $n=3,20$, and 22, respectively) treated in one institution between 2006 and 2017. All patients were progressive after systemic chemotherapy and had received at least two lines of platinum-containing combinations; three had undergone third-line and one patient fourth-line therapies. Previously given drugs contained combinations of cisplatin or carboplatin with paclitaxel, caelyx, treosulfan, or bevacizumab. The minimum chemotherapy-free interval before the start of abdominal perfusion therapy was 4 weeks. Performance status was ECOG 1 (7 patients), ECOG 2 (13 patients), and ECOG 3 (25 patients). Forty one patients $(91 \%)$ had peritoneal carcinosis and according to MRI, four patients had no evidence of peritoneal carcinosis. Progression of peritoneal carcinosis was classified by the affection of either two quadrants (11 patients, 24\%) or four quadrants (30 patients, 67\%). Seventeen patients had additional liver metastases, and three patients had metastases on the spleen. Lymphatic metastases were detected in 13 patients; one of them was inguinal, and in one case was mediastinal. Median number of metastatic locations (peritoneum considered as one location) was two. Surgery as a primary measure after progression was considered as unfeasible in all cases.

Investigations were performed in compliance with the principles of good clinical practice outlined in the Declaration of Helsinki and federal guidelines, and had approval by the Institutional Review Committee. Informed consent was obtained from each participant or participant's guardian. Patients were required to be $>$ 18 years of age and have an ECOG performance status $\leq$ 3. Exclusion criteria included cardiovascular diseases, as well as uncontrolled diabetes or serious infectious diseases. The leucocyte count had to be no less than $2500 / \mu 1$, but more importantly should not show a declining trend before start of the therapy. The same applied to the thrombocyte count, with a threshold of no less than $100,000 / \mu 1$.

\section{Isolated Hypoxic Abdominal Perfusion}

Under general anesthetic, the arteria and vena femoralis were exposed and tied with tourniquets. An arterial stop-flow catheter (PfM, Cologne, Germany and Dispomedica, Hamburg, Germany) was placed over the arterial transverse incision under X-ray monitoring, with the balloon above the diaphragm. The venous stop-flow catheter was secured with a purse-string suture and inserted through a stab incision. The balloon was placed in the inferior vena cava above the confluence of the right hepatic vein and below the right atrium. After blocking the balloons and checking the position with contrast medium, the balloons were unblocked again and both thighs were initially blocked with pneumatic cuffs. Under temporary hyperoxygenation, the chemotherapeutic agents were then applied through the aortal catheter as a bolus and both balloon catheters were immediately blocked again (Fig. 1). It is mandatory to inject the drugs under prior hyperoxygenation directly before balloon-blocking. The subsequent therapy was conducted for 15 min under hypoxic conditions in which mitomycin and adriamycin unlike other chemotherapeutics produce increased tumor toxicity [28]. Only the effect of cisplatin is not influenced by the $\mathrm{pH}$ value. Leakage monitoring of the isolated abdominal perfusion segment was not required; as after 15 min of hypoxic perfusion, the stop-flow balloon catheters and femoral pressure cuffs were unblocked and the systemic chemofiltration started immediately. Chemofiltration is maintained up to a substitution volume of at least 41 of filtrate. After completing chemofiltration, the catheters were removed and the artery and vein sutured successively.

\section{Treatment and Drug Regimen}

The isolated abdominal hypoxic perfusion was conducted in 4 cycles at 4 -week intervals. The cytostatic drugs used were cisplatin, adriamycin, and mitomycin. Using a bolus injection in the abdominal aorta at the diaphragm level, the maximum intra-arterial total dose of CDDP was $70 \mathrm{mg}$ at one shot. For $\mathrm{ADM}$, the maximum dose was $50 \mathrm{mg}$ and for MMC $20 \mathrm{mg}$ at one shot. For a patient of $70 \pm 5 \mathrm{~kg}$, the overall dose of CDDP was $60 \mathrm{mg}, \mathrm{ADM} 40 \mathrm{mg}$, and MMC $20 \mathrm{mg}$. Due to their intraaortal administration, the cytostatic drugs were not dosed by body weight for extremely obese patients.

Leucocytes and thrombocytes were monitored weekly after every treatment; while decreasing close to the lowest nadir, checks were carried out every 2 days until the blood count started to reemerge.

\section{Response Evaluation and Statistical Analysis}

The main endpoint of the trial was quality of life, overall survival, and clinical response rates. Tumor responses were visualized and assessed in accordance with Response 
Fig. 1 Isolated hypoxic abdominal perfusion via a femoral access. The balloon catheters are positioned beneath the diaphragm and connected with an extracorporeal roller pump

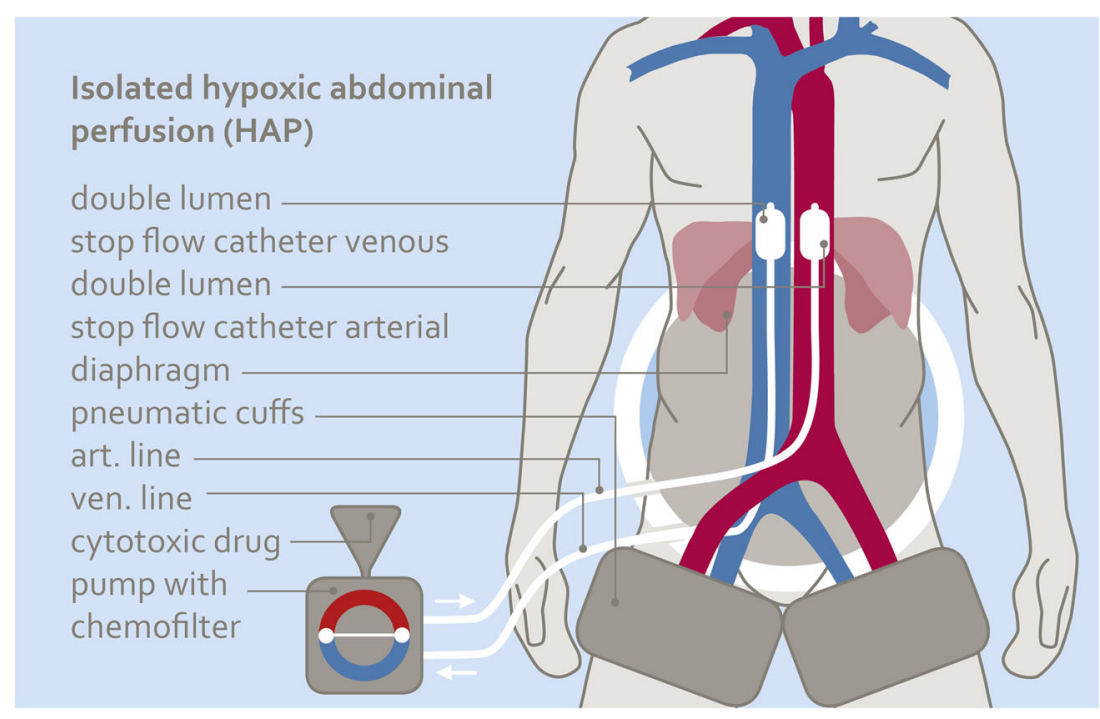

Evaluation Criteria in Solid Tumors (RECIST version 1.1) at 2 to 4 weeks after every second treatment cycle. Responses were evaluated by CT, magnetic resonance imaging (MRI), and positron emission tomography (PET). Plasma levels of the tumor marker CA 12-5 were determined before any treatment. The extent of residual disease, the degree of response in ascites, as well as the course of the tumor marker CA 12-5 were evaluated and compared. A reliable pain relief was noted if pain was controlled by < $50 \%$ analgesic administration 20 days after treatment. Adverse events were assessed according to the common terminology criteria for adverse events of the National Cancer Institute. Quality of life was evaluated by a patient questionnaire according to RCT institutional QOL approvement and compared to questionnaires regarding previous systemic chemotherapy treatments. Statistics have been calculated with $95 \%$ confidence limits, or as specified, survival times were estimated using the Kaplan-Meier product limit estimator. Survival times were stratified according to clinical variables that may affect survival, and log-rank tests were used to verify significance. Statistical analyses were performed by using the institutional research software, version 28.5.14.

\section{Blood Sampling for Cisplatin and Mitomycin C Plasma Concentration Measurements}

A series of cisplatin (CDDP) and Mitomycin C (MMC) plasma concentration measurements have been investigated. Blood samples derive from a patient with ovarian cancer and peritoneal carcinosis during and after hypoxic abdominal perfusion with CDDP and MMC. Arterial and venous drug concentrations, originating from the tumor region, were measured at minute 1,2 , and 3 , respectively, following measurements at 2-min intervals.

\section{Results}

\section{Response and Survival Rates}

The median progression-free survival (PFS) of all 45 patients was 6.9 months; the median overall survival was 11.3 months. The median survival rate of pretreated platinum-refractory patients at FIGO stage IIIB/C was 12.3 months, and at stage IV was 9.8 months (Fig. 2) $(p<0.05)$. Overall survival in FIGO III and IV together is $37.8 \%$ at 1 year, $18.3 \%$ at 2 years, stays $18.3 \%$ at 3 years, and $9.2 \%$ at 4 years. Time measurements started after completing multiple lines of standard chemotherapy, diagnosis of recurrent or progressive disease, and start treatment with hypoxic abdominal perfusion therapy. According to tumor marker 12-5, complete response and partial response have been achieved in $17.8 \%$ and $55.6 \%$ of all patients, respectively. CT or MRI visualization showed complete response and partial response in 4.1 and $54.1 \%$ of the cases (Fig. 3), respectively.

\section{Arterial and Venous Concentrations of CDDP and MMC}

During and after 15 min of hypoxic abdominal perfusion, drug concentrations were measured from plasma deriving from the inferior vena cava and from the aorta, both inside the perfusion area, and additionally from a peripheral vein outside the perfusion area (Fig. 4).

\section{Toxicity}

Bone-marrow suppression was low for 43 patients and ranged between WHO grade 1 and 2 . Two patients in 
Fig. 2 Observational survival rates of 45 advanced staged, heavily pretreated, recurrent ovarian cancer patients on hypoxic abdominal perfusion chemotherapy. Three patients were staged FIGO IIIB, 20 patients were stage IIIC, and 22 patients were staged IV. Survival times were estimated using the Kaplan-Meier product limit estimator, and follow-up for surviving patients was minimum 18 months; median follow-up was 26 months

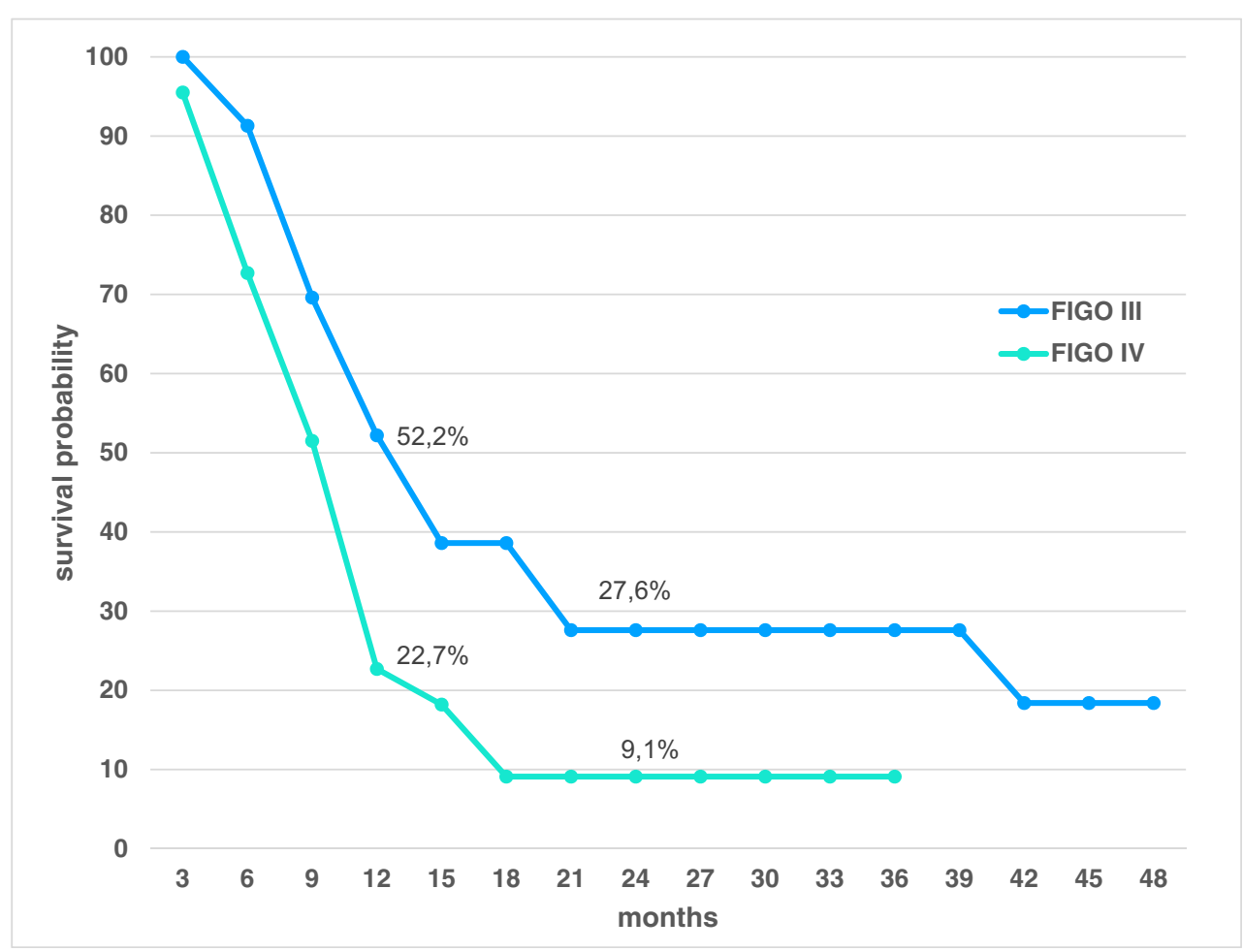

poor general condition following third- and fourth-line chemotherapy had leucopenia and thrombocytopenia WHO grade 3. Neutropenic fever at WHO grade 4 toxicity was never observed. Fatigue syndrome never occurred before the third day post-therapy and, if it occurred, it was always associated with an increase in both $\mathrm{LDH}$ and the tumor marker CA 12-5. Hypodense areas seen simultaneously in CT scans were identified as an expression of post-therapeutic tumor necrosis. All of these syndromes were observed during the first week after isolated perfusion. Mild renal toxicity with slight transient elevation of creatinine was observed in seven $(15.6 \%)$ patients. Neuropathy in terms of hand-foot syndrome was never observed after isolated perfusion with chemofiltration.

\section{Quality of Life}

Complete or partial response on clinical symptoms was noted among $36.3 \%$ of all patients in stages IIIB/C and IV. Stable disease was achieved in $51.1 \%$ of patients. Complete disappearance of ascites was observed in $30 \%$

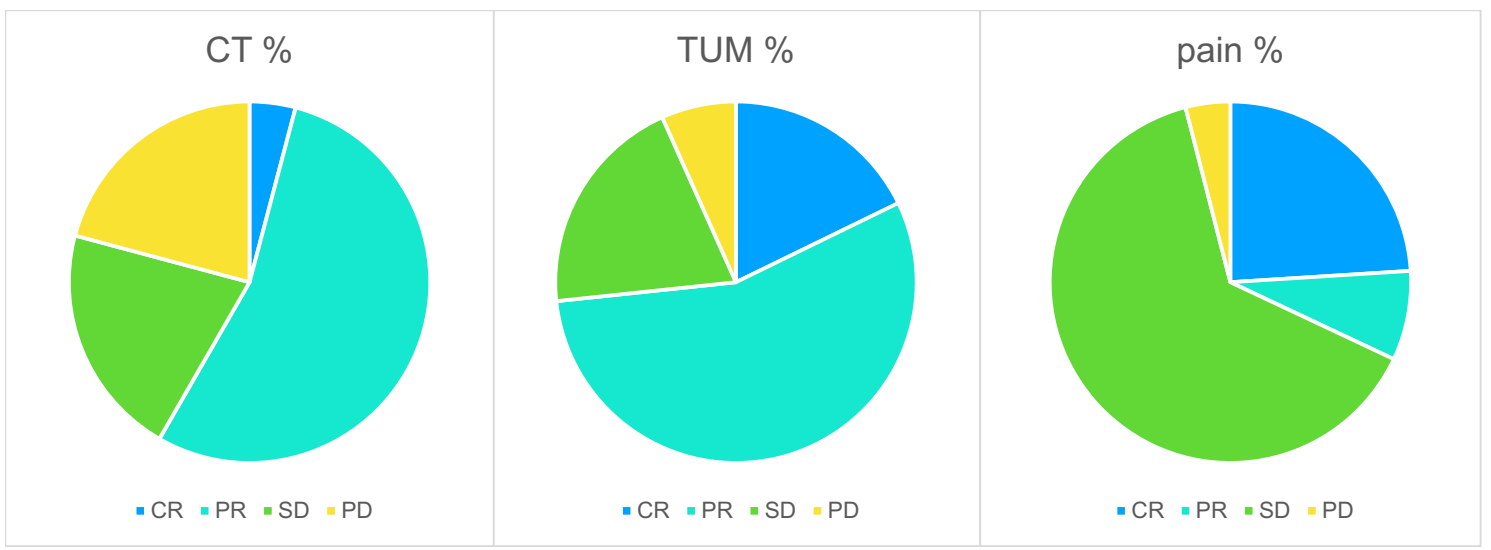

Fig. 3 Response rates of 45 advanced staged, heavily pretreated, recurrent metastatic ovarian cancer patients on hypoxic abdominal perfusion chemotherapy. Response classification was complete response
(CR), partial response (PR), stable disease (SD), and progressive disease (PD). Responses of a clinical benefit (CR, PR, SD) endured at least 8 weeks 
Fig. 4 Drug concentration levels of cisplatin (a) and MMC (b) in the tumor supplying arteria (violet), the tumor draining vein (blue), and the peripheral vein (green) during and after a hypoxic abdominal perfusion.

Measurements inside the perfusion circuit (arterial and venous) have been made at minute 1,2 , and 3 , and then every 2 min until 35 min after drug injection. Drug levels in the peripheral vein have been measured at minute 1,5 , and after releasing of perfusion balloons at minute 15 . Given drug regimen was $50 \mathrm{mg}$ cisplatin and $20 \mathrm{mg}$ mitomycin as a bolus injection. Both charts derive from the same perfusion event
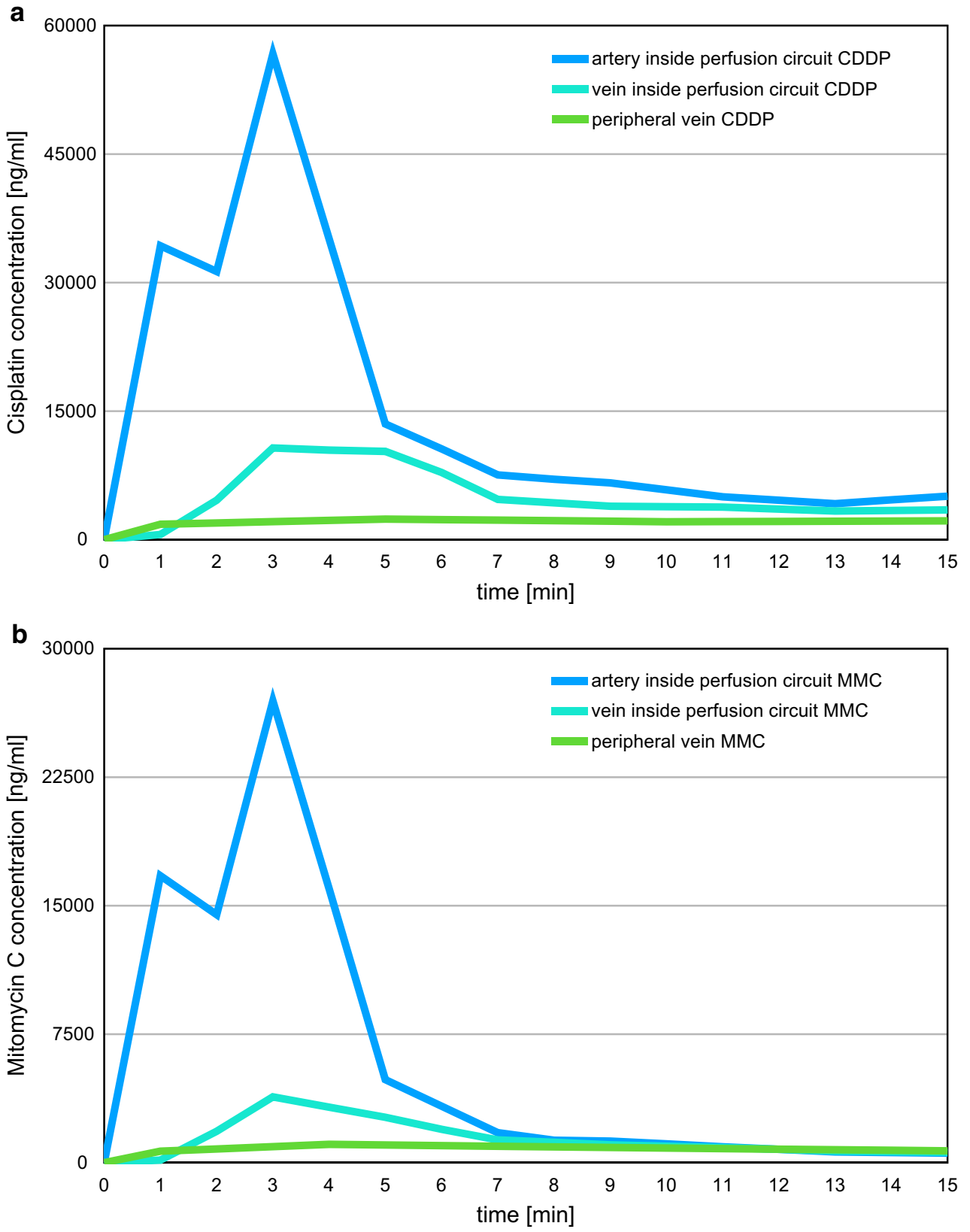

of patients after only two perfusions; $43 \%$ of patients reported a substantial reduction of abdominal pressure and fluid volume and a considerable improvement in general wellbeing. As shown in Fig. 5, patients perceived hypoxic abdominal perfusion (HAP) therapy less stressful than conventional chemotherapy. A patient questionnaire showed decrease in adverse events for the symptoms nausea, hair loss, diarrhea, mucositis, fatigue, exhaustion, weight loss, and anorexia. The mean decrease over all collected data for HAP was 1.86 (confidence interval, with $91 \%$ CI 0.5 ). A definite decrease in pain, which was controlled by $<50 \%$ analgesic administration 20 days after treatment, is reported for $30 \%$ of patients with advanced ovarian carcinoma.

\section{Discussion}

The limiting factor of augmented systemic drug exposure always had been the increasing toxicity such as neuropathy with hand-foot syndrome, neutropenia, or fatigue to the point of exhaustion [9-15]. Since mortality rates of ovarian cancer remain high and have hardly changed in the last three decades, more recent treatment options are being tested in the context of molecular biology research. Based on the angiogenetic properties of ovarian cancer with induction of vascularization, there has been an expectation that targeted treatments could achieve a restriction of tumor blood supply, higher response rates, and improved survival times, while sparing healthy 


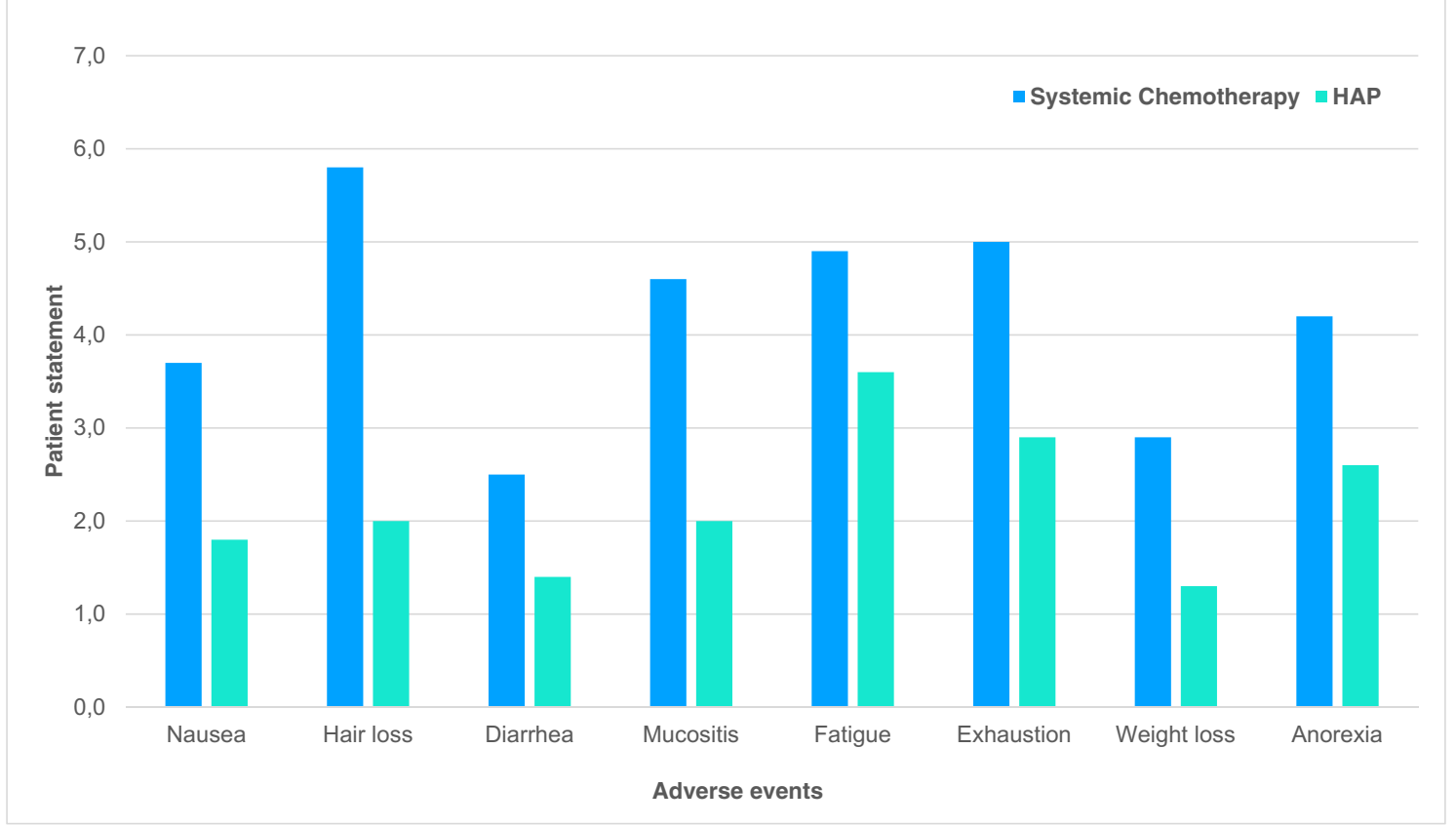

Fig. 5 Adverse effects after systemic chemotherapy (green) and hypoxic abdominal perfusion (HAP blue). Ovarian cancer patients filled in a questionnaire about adverse effects after chemotherapy. Each possible adverse effect is scaled on a spectrum from 1 to 6 for increasing severeness of side effects. Mean reduction of adverse events was 1.86 points for hypoxic abdominal perfusion compared to systemic chemotherapy, that patients perceived at an earlier treatment period (confidence interval for 1.86 (with $91 \%$ CI 0.5$)$ tissue [24]. Apart from prolonging PFS in ovarian cancer, targeted antiangiogenic therapies occasionally cause severe collateral damage in terms of high blood pressure, hemorrhages, protein urea, cardiotoxicity, and gastrointestinal toxicity with spontaneous perforations [23]. In a trial on 32 patients pretreated with multiple chemotherapy regimens, positive results were achieved with bevacizumab [24]. The median survival time was 6.9 months, with a median PFS of 5.5 months. With the limitation that the patient groups are not necessarily exactly comparable, these data do not reach the median survival time of 11.3 months at a PFS of 6.9 months reported here, following hypoxic isolated abdominal perfusion.

Prolonging life while maintaining or improving quality of life should be the basic requirement for any cancer treatment. No other fundamental necessity for any treatment to be recommended should actually apply [25]. In a comparative study, we looked at patients who were in progression following systemic chemotherapy and were then treated with regional chemotherapy [29]. Patients were asked to complete a questionnaire on the severity of the most common side effects, comparing their previous systemic therapy and the regional chemotherapy. Results showed that regional chemotherapy resulted in side effects that were perceived as less severe than the ones derived from systemic chemotherapy.

Surgical tumor debulking with the aim of complete cytoreduction is intended to prolong progression-free survival even in the case of advanced diseases, but it is ultimately limited to early stages, in which the desired curative removal of all lesions is still possible [30, 31]. At clinical stage IV, there is no difference in overall survival, no matter what treatment, chemotherapy, or primary cytoreductive surgery (PCS) was used. The administration of hyperthermic intra-peritoneal chemotherapy (HIPEC) has been considered another option of regional chemotherapy. The rationale behind is that after exposure of all peritoneal surfaces, the direct contact with hyperthermic chemotherapy generates a higher tissue uptake in residual tumors which is usually limited down to a depth of 1 to $2-\mathrm{mm}$ tumor thickness [32]. In contrast, the depth of penetration with intra-arterial therapy is homogenous throughout the entire tumor tissue, depending on the blood supply of the tumor. Metastases from ovarian cancer are well-vascularized and usually respond even in case of bulky lesions. So far, there have been studies with HIPEC for ovarian cancer in therapy for primary and for recurrent disease [33]. Particularly because of high morbidity and side effects, HIPEC was not recommended as a standard therapy for ovarian cancer [34, 35]. Only recently, however, two randomized studies showed that the addition of HIPEC to cytoreductive surgery resulted in longer survival than surgery alone. Hypoxic abdominal perfusion on the other hand is not associated with relevant toxicity, and quality of life is not impaired. It is not primarily performed in operable cases, appropriate for cytoreduction, but in systemically pretreated, advanced non-operable patients.

Presumably, advances in the treatment of various types of cancer such as ovarian, colorectal, or testicular carcinoma 
correlate extensively with the chemoresistance of tumor stem cells. While in the recent past, the cure rates of testicular carcinoma and even stage III colorectal carcinoma have increased significantly; the cure rates or clinical progress of ovarian carcinoma have remained unchanged, at between approx. $12 \%$ and $14 \%$ [36]. This may be related to the low response rate of epithelial ovarian carcinoma stem cells. An increase in overall survival in tumors in general may be due to suppression of the non-stem cell proportion of the tumor. It could also explain why further chemotherapy following recurrences brings about renewed remission and may even prolong life. Due to the existence of the problem of chemoresistant stem cells, these patients have limited therapeutic options and limited prospects for an improvement in prognosis.

Isolated perfusion therapy for bulky disease provides an option to a limited group of patients with advanced bulky disease, to achieve downsizing with operability, in the form of radical cytoreduction. In addition to stage IIIC, this would also apply to stage IV, where conventionally, primary cytoreductive surgery (PCS) has not provided any advantages in the past. The basic principle for increasing the cytostatic effect in the target area is the primary passage through the arterial blood supply with high cytostatic drug concentration, to increase the area under the curve (AUC). A high-concentration passage of the cytostatic in the form of a protracted bolus over 4 to $7 \mathrm{~min}$ results in an enhanced first-pass extraction, and based on experience, generate increased tumor necrosis even within the first few days after treatment [37-40]. In order to increase the local cytotoxicity without a significant increase in the overall dosage, the chemotherapeutic agent mitomycin (MMC) and adriamycin (ADM) were selected for perfusion under hypoxic conditions, which, as BA. Teicher described for the first time, produce multiple degrees of their normal cytotoxicity under hypoxic conditions [28]. Thus, the totally administered bonemarrow toxic dose can be kept low. Cisplatin (CDDP) activity is independent of oxygen conditions. Due to the increased cytostatic exposure with isolated perfusion techniques, it is possible to break through chemoresistance in platinum-refractory tumors. The extent to which this applies to tumor stem cells is unknown, but it could apply to initially well-advanced G3 tumors in the case of a few long-term surviving patients following regional chemotherapy. It is particularly remarkable that the survival curves, both in the chemoresistant stage IIIBC as well as IV, do not end abruptly after the median but show a survival rate of $18 \%$ and $9 \%$ even 3 years after therapy. "A focus on the tail of the survival curves, yields a distinct perspective on the benefit of anticancer therapies and prioritizes therapies with potential to profoundly alter the natural history of disease, even if uncommonly" [41]. This is an important aspect with a selective cohort of patients lifting the "tail" of the survival curve and thus enlarging the area under the curve.

Another important aspect in locally highly concentrated chemotherapy is the lowering of systemic cytostatic exposure by simultaneous chemofiltration [26]. Further improvement in quality of life is achieved in addition to systemic detoxification, through the rapid and high percentage decline in ascites. Seventy three percent of patients reported dramatic relief of their abdominal pain and discomfort. Quality of life should be a priority factor in tumor treatment, especially because newer treatment options, despite some advances, often only lead to slight increases in PFS or overall survival, at the expense of considerable toxicity [42]. As such, the approach of hypoxic abdominal perfusion warrants further investigation in selected patients with ovarian cancer not amenable to surgical resection. In the present situation, there was no control group of heavily pretreated patients available for repeated conventional chemotherapy. Therefore, the pretreated patients receiving isolated perfusion therapy, which is well tolerated due to chemofiltration, serve as their own control. A phase-III study, which investigates HAP versus systemic chemotherapy in non-pretreated patients, could provide information about future treatment options to be adopted.

\section{Conclusion}

Isolated abdominal perfusion under hypoxic conditions for advanced, recurrent, and platinum-refractory ovarian cancer can be an option for multiply pretreated, recurrent ovarian cancer with peritoneal carcinosis, even if drug resistance is existent. Profit in terms of high response rates is achieved by increased tumor toxicity of adriamycin and mitomycin under hypoxic conditions. Due to subsequent chemofiltration, systemic drug exposure is reduced and toxic side effects kept low. Quality of life generally is reported mostly unaffected.

Acknowledgments The authors wish to acknowledge Giuseppe Zavattieri and Rita Schlaf for their help and assistance in preparing the manuscript and statistics used in this report.

\section{Compliance with Ethical Standards}

Conflict of Interest The authors declare that they have no conflict of interest.

Ethical Approval All procedures performed in studies involving human participants were in accordance with the ethical standards of the institutional research committee and with the 1964 Helsinki declaration and its later amendments or comparable ethical standards.

Informed Consent Informed consent was obtained from all individual participants included in the study. 
Abbreviations ECOG, Eastern Cooperative Oncology Group; $C T$, computed tomography; FIGO, Fédération Internationale de Gynécologie et d'Obstétrique; $C A$, Carbohydrate antigen; $M M C$, mitomycin $\mathrm{C}$; $C D D P$, Cisplatin; $P F S$, Progression-free survival; WHO, World Health Organization; $O S$, Overall survival; $L D H$, Lactate dehydrogenase; $P C S$, Primary cytoreductive surgery; $N A C T$, Neoadjuvant chemotherapy; $H I P E C$, Hyperthermic intra-peritoneal chemotherapy; $A U C$, Area under the curve; TUM, Tumor marker; $A D M$, Adriamycin

Open Access This article is distributed under the terms of the Creative Commons Attribution 4.0 International License (http:// creativecommons.org/licenses/by/4.0/), which permits unrestricted use, distribution, and reproduction in any medium, provided you give appropriate credit to the original author(s) and the source, provide a link to the Creative Commons license, and indicate if changes were made.

\section{References}

1. Meyer LA, Cronin Angel M, Sun Charlotte C et al (2016) Use and effectiveness of neoadjuvant chemotherapy for treatment of ovarian cancer. J Clin Oncol 34:3854-3863

2. Vergote I, Trope CG, Amant F et al (2010) Neoadjuvant chemotherapy or primary surgery in stage IIIC or IV ovarian cancer. N Engl J Med 363:943-953

3. Vergote I, Van Nieuwenhuysen E, Vanderstichele A (2016) How to select neoadjuvant chemotherapy or primary debulking surgery in patients with stage IIIC or IV ovarian carcinoma. J Clin Oncol 34: 3827-3828

4. Gore ME, Fryatt I, Wiltshaw E (1990) Treatment of relapsed carcinoma of the ovary with cisplatin or carboplatin following initial treatment with these compounds. Gynecol Oncol 36:207-211

5. Markman M, Rothman R, Hakes T, Reichman B, Hoskins W, Rubin S, Jones W, Almadrones L, Lewis JL Jr (1991) Secondline platinum therapy in patients with ovarian cancer previously treated with cisplatin. J Clin Oncol 9:389-393

6. Ozols RF (1997) Treatment of recurrent ovarian cancer: increasing options - "recurrent" results. J Clin Oncol 15:2177-2180

7. Markman M, Bookman MA (2000) Second line treatment of ovarian cancer. Oncologist 5:26-35

8. Gore M, Mainwaring P, A'Hern R et al (1998) Randomized trial of dose-intensity with single-agent carboplatin in patients with epithelial ovarian cancer. London Gynaecological Oncology Group. J Clin Oncol 116:2426-2434

9. Jodrell DI, Egorin MJ, Canetta RM, Langenberg P, Goldbloom EP, Burroughs JN, Goodlow JL, Tan S, Wiltshaw E (1992) Relationships between carboplatin exposure and tumor response and toxicity in patients with ovarian cancer. J Clin Oncol 10:520 528

10. Levin L, Hryniuk WM (1987) Dose intensity analysis of chemotherapy regimens in ovarian carcinoma. J Clin Oncol 5:756-767

11. McGuire WP, Hoskins WJ, Brady MF et al (1995) Assessment of dose-intensive therapy in suboptimally ovarian cancer: a Gynecologic Oncology Group study. J Clin Oncol 13:1589-1599

12. Markman M, Liu PY, Wilczynski S, Monk B, Copeland LJ, Alvarez RD, Jiang C, Alberts D (2003) Phase III randomized trial of 12 versus 3 months of maintenance paclitaxel in patients with advanced ovarian cancer after complete response to platinum and paclitaxel-based chemotherapy: a Southwest Oncology Group and Gynecologic Oncology Group trial. J Clin Oncol 21:2460-2465

13. Markman M, Liu PY, Moon J, Monk BJ, Copeland L, Wilczynski S, Alberts D (2009) Impact on survival of 12 versus 3 monthly cycles of paclitaxel $\left(175 \mathrm{mg} / \mathrm{m}^{2}\right)$ administered to patients with advanced ovarian cancer who attained a complete response to primary platinum-paclitaxel: follow-up of a Southwest Oncology Group and Gynecologic Oncology Group phase 3 trial. Gynecol Oncol 114: 195-198

14. Omura GA, Brady MF, Look KY, Averette HE, Delmore JE, Long HJ, Wadler S, Spiegel G, Arbuck SG (2003) Phase III trial of paclitaxel at two dose levels, the higher dose accompanied by filgrastim at two dose levels in platinum-pretreated epithelial ovarian cancer: an intergroup study. J Clin Oncol 21:2843-2848

15. Thigpen JT (1997) Dose-intensity in ovarian carcinoma: hold, enough? J Clin Oncol 15:1291-1293

16. Grenman S, Wiklund T, Jalkanen J et al (2006) A randomised phase III study comparing high-dose chemotherapy to conventionally dosed chemotherapy for stage III ovarian cancer: the Finnish Ovarian (FINOVA) study. Eur J Cancer 42:2196-2199

17. Möbus V, Wandt H, Frickhofen N, Bengala C, Champion K, Kimmig R, Ostermann H, Hinke A, Ledermann JA (2007) Phase III trial of high-dose sequential chemotherapy with peripheral blood stem cell support compared with standard dose chemotherapy for first-line treatment of advanced ovarian cancer: intergroup trial of the AGO-Ovar/AIO and EBMT. J Clin Oncol 25:4187-4193

18. Fung MF, Johnston ME, Eisenhauer EA et al (2002) Chemotherapy for recurrent epithelial ovarian cancer previously treated with platinum - a systematic review of the evidence from randomized trials. Eur J Gynaecol Oncol 23:104-110

19. du Bois A, Weber B, Rochon J, Meier W, Goupil A, Olbricht S, Barats JC, Kuhn W, Orfeuvre H, Wagner U, Richter B, Lueck HJ, Pfisterer J, Costa S, Schroeder W, Kimmig R, Pujade-Lauraine E, Arbeitsgemeinschaft Gynaekologische Onkologie, Ovarian Cancer Study Group, Groupe d'Investigateurs Nationaux pour l'Etude des Cancers Ovariens (2006) Addition of epirubicin as a third drug to carboplatin-paclitaxel in first-line treatment of advanced ovarian cancer: a prospectively randomized gynecologic cancer intergroup trial by the Arbeitsgemeinschaft Gynaekologische Onkologie Ovarian Cancer Study Group and the Groupe d'Investigateurs Nationaux pour l'Etude des Cancers Ovariens. J Clin Oncol 24: $1127-1135$

20. Spiliotis J, Halkia E, Lianos E, Kalantzi N, Grivas A, Efstathiou E, Giassas S (2015) Cytoreductive surgery and HIPEC in recurrent epithelial ovarian cancer: a prospective randomized phase III study. Ann Surg Oncol 22:1570-1575

21. van Driel WJ, Koole SN, Sikorska K, Schagen van Leeuwen JH, Schreuder HWR, Hermans RHM, de Hingh IHJT, van der Velden J, Arts HJ, Massuger LFAG, Aalbers AGJ, Verwaal VJ, Kieffer JM, van de Vijver KK, van Tinteren H, Aaronson NK, Sonke GS (2018) Hyperthermic intraperitoneal chemotherapy in ovarian cancer. $\mathrm{N}$ Engl J Med 378:230-240

22. Yi S, Zeng L, Kuan Y et al (2017) Antiangiogenic drugs used with chemotherapy for patients with recurrent ovarian cancer: a metaanalysis. Onco Targets Ther 10:973-984

23. Stone RL, Sood AK, Coleman RL (2010) Collateral damage: toxic effects of targeted antiangiogenic therapies in ovarian cancer. Lancet Oncol 11:465-475

24. Monk BJ, Han E, Joseph-Cowen CA et al (2006) Salvage bevacizumab (rhuMABVEGF)-based therapy after multiple prior cytotoxics regimens in advanced refractory epithelial ovarian cancer. Gynecol Oncol 102:140-144

25. Cannistra SA (2004) The ethics of early stopping rules: who is protecting whom? J Clin Oncol 22:1542-1545

26. Aigner KR, Gailhofer S (1993) High dose MMC: aortic stopflow infusion (ASI) with versus without chemofiltration: a comparison of toxic side effects (abstract). Reg Cancer Treat 6(Suppl 1):3-4

27. Aigner KR, Tonn JC, Hechtel R et al (1983) Die intraarterielle Zytostatikatherapie mit venöser Filtration im halboffenen System. Onkologie 6:74-76 
28. Teicher BA, Lazo JS, Sartorelli A (1981) Classification of antineoplastic agents by their selective toxicities toward oxygenated and hypoxic tumor cells. Cancer Res 41:73-81

29. Aigner KR, Knapp N (2016) Toxicity profiles with systemic vs. regional chemotherapy. In: Aigner KR, Stephens FO (eds) Induction chemotherapy - systemic and locoregional, Second edn. Springer Verlag, Berlin, pp 497-506

30. Crawford SC, Vasey PA, Paul J, Hay A, Davis JA, Kaye SB (2005) Does aggressive surgery only benefit patients with less advanced ovarian cancer? Results from an international comparison within the SCOTROC-1 trial. J Clin Oncol 23:8802-8811

31. Kehoes S, Hook J, Nankivell M et al (2015) Primary chemotherapy versus primary surgery for newly diagnosed advanced ovarian cancer (CHORUS): an open-label randomized, controlled, noninferiority trial. Lancet 386:249-257

32. van de Vaart PJ, van der Vange N, Zoetmulder FA et al (1998) Intraperitoneal cisplatin with regional hyperthermia in advanced ovarian cancer: pharmacokinetics and cisplatin-DNA adduct formation in patients and ovarian cancer cell lines. Eur J Cancer 34:148-154

33. Bijelic L, Jonson A, Sugarbaker PH (2007) Systematic review of cytoreductive surgery and heated intraoperative intraperitoneal chemotherapy for treatment of peritoneal carcinomatosis in primary and recurrent ovarian cancer. Ann Oncol 18:1943-1950

34. Gore M, du Bois A, Vergote I (2006) Intraperitoneal chemotherapy in ovarian cancer remains experimental. J Clin Oncol 24:4528-4530

35. Harter P, Heitz F, du Bois A (2012) Surgery for relapsed ovarian cancer: when should it be offered? Curr Oncol Rep 14(6):539-543. https://doi.org/10.1007/s11912-012-0260-x

36. Lan H, Cronin KA, Johnson KA et al (2010) Improved survival time: what can survival cure models tell us about population-based survival improvements in late-stage colorectal, ovarian, and testicular cancer? Cancer 112:2289-2300

37. Stephens FO, Harker GJS, Crea P (1980) The intra-arterial infusion of chemotherapeutic agents as "basal" treatment of cancer: evidence of increased drug activity in regionally infused tissues. Aust NZ J Surg 50:597-602

38. Stephens FO (1988) Why use regional chemotherapy? Principles and pharmacokinetics. Reg Cancer Treat 1:4-10

39. Guadagni S, Clementi M, Valenti M, Fiorentini G, Cantore M, Kanavos E, Caterino GP, di Giuro G, Amicucci G (2007) Hypoxic abdominal stop-flow perfusion in the treatment of advanced pancreatic cancer: a phase II evaluational trial. Eur J Surg Oncol 33:72-7840

40. Stephens FO (2001) Induction (neo-adjuvant) chemotherapy: the place and techniques of using chemotherapy to downgrade aggressive or advanced localised cancers to make them potentially more curable by surgery and/or radiotherapy. Eur J Surg Oncol 27:627688

41. Hellmann M, Kris MG, Rudin CM (2016) Medians and milestones in describing the path to cancer cures: telling "tails". JAMA Oncol 2:167-168

42. Aigner KR, Stephens FO (2012) Guidelines and evidence-based medicine - evidence of what? EJCMO 4:(3). Available at: http:// www.slmoncology.com/Guidelines_and_Evidence_Based Medicine_Evidence_of_What_1,272.html. Accessed Sept 2012

Publisher's Note Springer Nature remains neutral with regard to jurisdictional claims in published maps and institutional affiliations. 